\title{
GLOBAL ISSUE BASED POWER GENERATION EXPANSION PLANNING FOR A POWER SYSTEM
}

\author{
G.Srinivasulu ${ }^{1}$, Y.V.NiranjanKumar ${ }^{2}$, K.V.Kishore ${ }^{3}$, P. Udai Kumar ${ }^{4}$ \\ ${ }^{1}$ Associate Professor, Dept of EEE, Narayana Engineering College: Nellore. India, gs_meghana@rediffmail.com \\ ${ }^{2,3,4}$ Assistant Professor Dept of Electrical \& Electronics Engineering, Narayana Engineering College: Nellore, India. \\ ${ }^{2}$ y.v.niranjankumar@gmail.com, ${ }^{3}$ k.v.kishore288@gmail.com, ${ }^{4}$ pennuballi.udaikumarreddy@gmail.com
}

\begin{abstract}
In This Project an global issue based power generation expansion planning model has been developed for optimization that considers the growth of fuel prices and its fluctuation, benefits of carbon-trading in generation expansion decision, power risks. The Developed model is applied to An Electric Power System for the future. In an electric power system, the electric demand has been running ahead of supply. In addition, of the growth of fuel price can affect the country economy. To minimize these problems, a multi-objective model preceded by electric demand forecasting is developed by considering the unit of power generation and investment cost, imported fuels and benefits of carbon trading, environmental impacts. A model has been developed in the MATLAB and is simulated to get the output of each power plant at a very nominal cost for building the power plants, production and fuel.
\end{abstract}

Keywords: electrical power generation, expansion, planning, Load forecasting, Optimizing, linear model

\section{INTRODUCTION}

Energy plays a vital role in every aspect, among the different types of energies available electrical energy is considered to be superior, as it is convenient, easy to control, cleanness, greater flexibility, can be effectively be transformed and it is very essential for better quality of the life.

It is being one of the important sectors in the economy. One of The study estimates that the output lost, due to a one-day power cut, is to be between $10 \%$ to $15 \%$ of that day's GDP. Potential losses from power disruption will increase in the future as the economy grows and the relative contributions of the industry and service sectors increase in the economy.

Power supply has special characteristics which make the service unique as compared to other types of industry. The end product has to be delivered instantaneously and automatically upon the consumer's need (Demand).Therefore, to meet electricity requirements for customers, planning of the electric sector is of great importance since the decisions to be taken involving the commitment of large resources, with potentially serious economic risks for the electrical utility and the economy as a whole.

Planning is part and parcel of the development planning, the main objective of this is to have a reduced cost scenario for the future (in long term) expansion of generation, transmission, and distribution to meet the energy demands for a fixed tenure with in the constrained limits (like technical, economical, energy policies, and environmental effect).
We are mainly concentrated to power generation expansion planning because it is clear fact that the cost incurred in the expansion of transmission line or distribution feeders are less when compared to that of the generation.

The generation expansion planning problem is defined as the problem of determining WHAT, WHEN, and HOW MUCH new generation units should be installed over a long-range planning horizon, to satisfy expected energy demand. This problem is a strategic planning problem for any country.

Generation expansion planning has historically addressed the problem of identifying ideal technology, expansion size, sitting, and timing of construction of new plant capacity in an economic fashion and in a manner that ensures installed capacity adequately meets projected demand growth. However, deregulation and current issues alter traditional generation expansion planning assumptions, models, and methods.

Now a day, the issue of global warming has great importance for the decision of power generation planning. Every nation is taking their own measures to minimize the global warming, like sharing the ultimate objective of the convention to stabilize atmospheric concentrations of greenhouse gases at a level that will prevent dangerous interference with the climate system.

Under these measures we have Kyoto mechanism, Other mechanism is Clean Development Mechanism (CDM).In early application of solutions, several methods have been proposed to solve the problem of generation expansion planning. The approaches have been focusing to minimize cost. This thesis is 
granted to profit maximization and cost minimization to be treated simultaneously for the optimization of power generation expansion planning with the incorporation of global issue.

\section{IDENTIFICATION OF PROBLEM}

According to International Energy Agency (IEA) reports, fossil fuels remain the dominant sources of primary energy worldwide accounting for more than three-quarters of the overall increase in energy use between 2010 and 2030. This results high amounts of $\mathrm{CO} 2$ emissions to the atmosphere and contributing adverse greenhouse effect.

To reduce the non-renewable energy generation plants, the rapid change to renewable energy sources is being unlikely implemented due to their high investment cost, availability, energy market, policy and regulatory frameworks established at national and international levels.

Therefore, non-renewable energy resources plants will continue to provide a large proportion of world's commercial energy, and making the world to think about carbon dioxide capture and storage to control $\mathrm{CO}_{2}$ emissions.In the recent IEA report, in the broad scientific view they have stated that with the increase in global average temperature above pre-industrial levels ought not to exceed $2^{\circ} \mathrm{C}$ by 2020 . For this, several governments have committed to provide funding support for demonstration and implementing projects to be responsible for reduction of $\mathrm{CO}_{2}$ emissions. To reduce global emissions, the engagement of developing countries through capacity building and mapping of carbon storage potential, is vital.

Nevertheless, the energy sector in our country is composed of three main sub-sectors: biomass, petroleum and Electricity. About $80 \%$ of the energy demand in our country comprises of wood as fuel. As a result of which the availability of the wood in the stocks are declined drastically year by year. This results in environmental degradation and global warming.

Some of the developing nations are losing benefits from carbon trading. The dung consumed for energy demand satisfaction is also significant amount; the estimations suggest that the reduction in agricultural productivity, another relevant issue is uncertainty in fuel price and its growth trend. This is significant to affect the country economic development.

The energy demand is expected to be grown much more for the next five and above years due to population growth, social and economical developments. Thus, following the previous trends of generation expansion planning will not satisfy the electricity demand.

To consider all the aspects mentioned above is the desire of any decision maker in generation expansion planning, and that is the motivation for the work presented in this thesis.

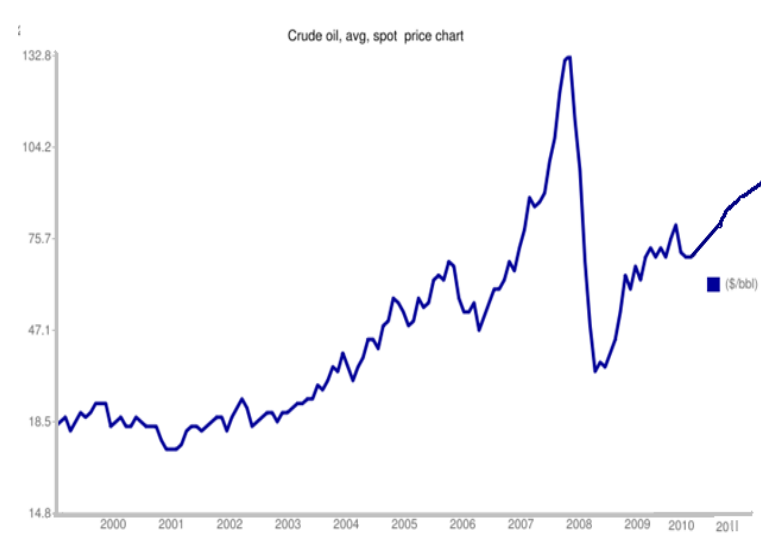

FIG-1 REPORT FROM IEA ON THE ENERGY USE FOR 2010-30

\section{AIMS AND OBJECTIVES}

In this paper a feasible model is developed which is used to solve the problem of electric power generation expansion and planning for an electric power system for a tenure of 10 years in the mere future, it has the following special

$>$ For the analysis of demand forecasting of the nation for about 10 years in the future.

$>$ Optimization of selected objectives and constraints those are considered in the power generation expansion and planning.

$>$ Developing of mathematical model and formulating for the chosen object and constraints in the electric power system.

$>$ For the developed model, solution technique has to be identified and implemented.

$>$ Simulating of proposed model by MATLAB and judge the performance of the model.

\section{MATHEMATICAL MODELLING}

Multiple, conflicting and disproportionate objectives are the be involved in the power generation expansion and planning. Modelling is more realistic by considering distinct and different objectives rather than considering a single objective function and the planning must consider the restrictions and must develop concepts and structures which are technically and economically sound.

The mathematical modelling is described by considering the decision variables, constraints and objective functions.In the power generation expansion planning several factors like Economics, socio-cultures, institutional policies, environmental impacts, technology and space are factors to be taken into consideration while modeling

Among them, focusing is done on economics (investment and generation cost), environmental impacts, carbon trading and energy imported. 


\section{A) OBJECTIVE FUNCTION:-}

\section{1) Investment and Generation Cost:-}

Investment cost is due to total cost for building the power plants. It is approximately proportional to MW generated.Generation cost is the total annual cost incurred in power generation. It has three parts consisting fixed cost, semifixed and running cost.Investment and generation cost can be formulated by adding the investment cost of new generating units and generating cost of existing and new units.

$$
f_{1}=\sum_{p=1}^{M} I_{P}\left(g n_{p}\right)+\sum_{p=1}^{M} G_{p}\left(g e_{p}+g n_{p}\right)-(\text { (01) }
$$

\section{2)Environmental Impacts:-}

This issue contains the emissions of greenhouse gases, social impacts, and impact on wild life, air pollution, aesthetic, and land management. In this paper we are considering only the emission. Of the all emissions like $\mathrm{CO}_{2}, \mathrm{NX}_{\mathrm{O}}, \mathrm{SO}_{2}$, We are more concern about carbon dioxide emission.

$$
f_{2}=\sum_{p=1}^{M} E_{p}\left[g e_{p}+g n_{p}\right]---(02)
$$

\section{3) Cost of Imported Fuel:-}

It can be determined multiplying unit cost of imported fuel by amount of imported fuel (taking corresponding unit). The fuel can be coal, oil or natural gas.

$$
f_{3}=\sum_{k=1}^{F} V_{k}\left(\boldsymbol{u}_{k}\right)--(03)
$$

\section{4) Carbon Trading:-}

If the nation can capture carbon dioxide emission released by any other country to the atmosphere then it is the benefit for the nation, this is achieved by planting trees. An amount of the revenue can be determined by evaluating the plants which can be equivalent to the MWh electrical energy produced by generating units, and by finding out the estimated hectare coverage or ton of plants which are corresponding to energy supply. The amount obtained in US-DOLLOR is given by $R_{P}(\$ / M W h)=[T T(\$ / T O N)] /[E E(M W h / T O N)]$

$$
\text { ---- (04) }
$$

Replacing the woody fuel consumption by electricity gives revenu from the one MWh electric energy generated from power plants to be newly constructed.

$$
f_{44}=\sum_{p \in M} R_{p}\left(g n_{p}\right)-- \text { ( 05) }
$$

The maximization function $\mathrm{f}$ can be converted to minimization function multiplying by -1 . So, equation (05) is written as

$$
f_{4}=-f_{44}=-\sum_{p \in M} R_{p}\left(g n_{p}\right)--(06)
$$

These four objective functions need a way for solving the problem easily. On this base, the first, third and fourth objective problems are expressing the cost.

$$
\begin{aligned}
& f_{134}=f_{1}+f_{3}+f_{4} \\
& f_{134}=\sum_{p=1}^{M} I_{p}\left(g n_{p}\right)+\sum_{P=1}^{M} G_{p}\left(g e_{p}+g n_{p}\right)+\sum_{K=1}^{F} V_{k}\left(u_{k}\right)-\sum_{P=1}^{M} R_{p}\left(g n_{p}\right)
\end{aligned}
$$

Here equation (02) is not the cost based function so we need to convert it into cost function then the total function becomes a single objective model, For that we need the multiply the equation with Emission cost then the equation becomes,

$$
f_{2}=\sum_{p=1}^{M} C E_{p}\left[g e_{p}+g n_{p}\right]--(08)
$$

The objective functions can be combined to single objective function.

$$
f_{1234}=f_{1}+f_{2}+f_{3}+f_{4--}(09)
$$

By rearranging we get the equation as ( 10 ) which is developed for the scenario -1

$$
f=\sum_{p e=1}^{m}\left(G_{p}+C E_{p}\right)\left(g e_{p}\right)+\sum_{p n=m+1}^{M}\left(I_{p}+G_{p}+C E_{p}-R_{p}\right)\left(g n_{p}\right)+\sum_{k=1}^{F} V_{k}\left(u_{k}\right)
$$

For the scenario-2 the equation would be of

$$
f=\sum_{p e=1}^{m}\left(G_{p}+C E_{p}\right)\left(g e_{p}\right)+\sum_{p n=m+1}^{M}\left(I_{p}+G_{p}+C E_{p}\right)\left(g n_{p}\right)+\sum_{k=1}^{F} V_{k}\left(u_{k}\right)
$$

\section{B) CONSTRAINTS:-}

1) Power Balance Equation:-

The total power generation from the plants is equal to sum of power consumed and lost.

$$
\sum_{p=1}^{M}\left[g e_{p}+g n_{p}\right]=D
$$

2) Generation Capacity of Each Unit type P:-

Operating Limits are imposed by the generation units

$$
\begin{aligned}
& g e_{p} \leq G E_{P} \\
& g n_{p} \leq G N_{p} ; p \leq M^{---(12 \mathrm{~A}) \&(12 \mathrm{~B})}
\end{aligned}
$$


3) Fuel Demand forEach Fuel Type K:-

Fuel used will be either from imported or if necessary from local markets.

$$
\sum_{p=1}^{M} W_{P}\left(g e_{p}+g n_{p}\right) \leq U_{k}+u k ; k \in F
$$

4) Non Negativity:-

No negative variables are permitted for the decisionvariables.

$$
g e_{p}, g n_{p}, u_{k} \geq 0, \forall p, k---(14)
$$

C) General Model:-

Let $f(x)$ be objective function then

$$
\mathrm{x}=(\mathrm{gp}, \mathrm{gn}, \mathrm{u})
$$

where $\mathrm{x}$ belongs to $\mathrm{X}$, which is a feasible space. The general model has $|\mathrm{M}|+|\mathrm{F}|$ variables and $3+|\mathrm{F}|$ constraints.

\section{D) LOAD FORCASTING:-}

Forecasting is one the major work in power generation expansion planning. This is done on the basis of economic and demographic status of the country. An energy demand forecast is a measurement and estimate of historic, current and projected patterns of energy demand for the place of interest.

There are different methods of forecasting load /energy demand which include trend analysis, end-use analysis and econometric analysis. Mainly econometric and trend analyses are used. Enduse analysis (where suitable) is also used in this thesis work.

In this thesis we are considering only the Domestic Demand Forecasting in this forecasting again there are two scenarios.

a) Stretched Forecasting (scenario -1):-

It is used by carbon trade consideration, in this the forecasting is based on the last trends of electric consumption, but the trend varies mainly because of the following reasons.

$\rightarrow$ The government future plan and the last trends of growth of GDP.

$\rightarrow$ Increased Domestic Residencies

$\rightarrow$ Urban House Hold areas are increased simultaneously

There energy usage is also increased.

\section{b) Target Forecasting (scenario-2) :-}

In this scenario, the forecasting is determined on the basis of the growth trend of number of customers and economic analysis.

$$
\begin{aligned}
& c u s_{t}=b_{0}+b_{1} * t \\
& \text { where, } \\
& \text { cust }=\text { numberof } \cos \text { tumersatyear' } t^{\prime} \\
& b_{1}=\frac{n^{*} \sum\left(t_{i} * c u s_{i}\right)-\left(\sum t_{i}\right) *\left(\sum c u s_{i}\right)}{n^{*} \sum\left(t_{i} * t_{i}\right)-\left(\sum t_{i}\right) *\left(\sum t_{i}\right)} \\
& b_{0}=\frac{\sum c_{u s}}{n}-\frac{b_{1} * \sum t_{i}}{n} \\
& \text { cusi } i=\text { numberof cos tumersatithyear } \\
& t i=i_{\text {thyear }} \\
& n=\text { numberofsamples }
\end{aligned}
$$

The electric demand of the sector is obtained by the below equation.

$$
\operatorname{demt}_{c}=\operatorname{avecon}_{c} * \mathrm{cust}_{t}
$$

Where ,

demt $_{c}=$ total demand by costumer category at year $\mathrm{t}$.

avecon $_{\mathrm{c}}=$ average consumption in costumer category

\section{SOLUTION TECHNIQUE}

By using some optimized techniques the set of designed parameters $x=\left\{x_{1}, x_{2}, x_{3}, \ldots, x_{n}\right\}$ are to be found , it is so called as optimal. This is nothing but the objective function $\mathrm{f}(\mathrm{x})$ to be minimized or maximized, that might be subjected to constraints, in the form of

Equality constraints $\mathrm{Gi}(\mathrm{x})=0$ (where $\mathrm{I}=1$..,me); Inequality constraints $\mathrm{Gi}(\mathrm{x}) \leq 0$ (where $\mathrm{i}=\mathrm{me}+1 \ldots \mathrm{m})$; Parameters bounds $\mathrm{xi}, \mathrm{xu}$

Where $\mathrm{x}=$ vector of length ' $\mathrm{n}$ ' desgin parameter

$\mathrm{F}(\mathrm{x})=$ objective function which returns a scalar,

$\mathrm{G}(\mathrm{x})=$ returns a vector of length ' $\mathrm{m}$ ' (includes equality And inequality constrained evaluation of $\mathrm{x}$ ).

Optimization is nothing but allocating of scarce resources to the best possible effect.These type of optimization process is utilized in resource allocation, scheduling, planning and decision making.etc.,

\section{METHODS OF OPTIMZATION:-}

\section{A) CLASSICAL METHODB) NUMERICAL METHOD}

In this paper we are proceeding with numerical method and this method again comprises of two different models 


\section{1) Linear Programming:-}

In this the objective function ' $\mathrm{f}$ ' which is to be minimized or maximized is linear. The design variable space is specified using only linear equalities and inequalities.

\section{2) Non-linear Programming:-}

It is similar to that of linear programming but the relationship has a non-linear form i.e. objective function, constraints are having non-linear parts.

In this paper we are developing the mathematical models in linear form, so the solution technique is obtained by linear programming method.

\section{ALGORITHM}

In this paper we are implementing medium-scale simplex algorithm because it is efficient to get the solution to have the minimum value.

Before applying the algorithm to get the solution we are making our objective function in the form of

$$
f(x)=C_{1} x_{1}+C_{2} x_{2}+\ldots \ldots+C_{n} x_{n--}(18)
$$

Which can be written in matrix form as $f(x)=C^{T} x \quad--(19)$ In this medium - scale linear programming simplex algorithm we have two phases. A) phase-1(start-up) B) phase-2(iterate). The algorithm applies in the phase-2, starting at the initial point from phase-1 to solve the original problem. At each iteration the algorithm checks for the optimal condition and stop if the current solution is optimal.

If the solution is not optimal the algorithm then

A) Chooses one variable (entering or leaving variable)

B) Update the current solution and the current objective value.

To implement this algorithm the call function of the solver is given as

$\left[\begin{array}{llll}x & f & \text { exit } & \text { flag }\end{array}\right.$ A,b,Aeq,beq,lb,ub,x0,options)(20)

That returns the value of objective function $f$ at the solution $x$, value of exit flag that describes the exit condition, a structure output that contains information about the optimization.

The right hand side of the solver is the input arguments that to be fed by the programmer and the left hand side of the solver is nothing but the output arguments we get whether the solution is optimized or not, if it is not optimized the reason why the program is terminated is displayed in terms of numerical values.

Linprog returns empty output arguments for the decision variable $x$ and value of objective function ' $\mathrm{f}$ ' if it detects infeasibility or unboundness. When the problem is unbounded, linprogreturns ' $\mathrm{x}$ ' and ' $\mathrm{f}$ ' in the unbounded directions.
In this linprogto maximize the objective function gives rise to a related problem, called the dual problem, where we seek to minimize the objective function. The two problems interact in an interesting way: every feasible solution to one problem gives rise to a bound on the optimal solution in the other problem.i.e.

$$
\text { Min } C^{T} \mathrm{x} \Leftrightarrow \quad \operatorname{Max~b}^{\mathrm{T}} \mathrm{y}--(21)
$$

A. $x \leq b \quad$ A. $y \leq \mathrm{c}$

$\{\mathrm{n}$, variables, $\mathrm{m}$ constraints $\} \quad\{$ mvariables, $\mathrm{n}$ constraints $\}$

Finally every Linear Programming falls into one of the three categories,

i) Infeasible ii) Unbounded iii) Has an Optimal Solution.

\section{RESULT AND DISCUSSION}

For the designed model which is in the linear model,linear programming has been used to obtain the result. In this paper solver of LP Simplex Algorithm built in MATLAB is used.

f01 and f02 stand for coefficients of objective functions for model-1 and model-2 respectively. A, Aeq,b, beq,lb and ub represent the matrix of inequality constraints, equality constraints, the vector of the right hand side of inequality constraints, the vector of equality constraints (annual demand), lower bunds of decision variables( zero matrix) and upper bounds of decision variables (maximum generation capacity of the power plants) respectively.

Result for model -1;-

fopt $1=3.7103 \mathrm{e}+009$

exitflag $=1$ output $=$ iterations: 9

algorithm: 'mediumscale: simplex'

message: 'Optimizationterminated.'

Result for model-2:-

fopt $2=3.1198 \mathrm{e}+009$

exitflag $=1$ output $=$ iterations: 4

algorithm: 'mediumscale: simplex'

message: 'Optimizationterminated.'

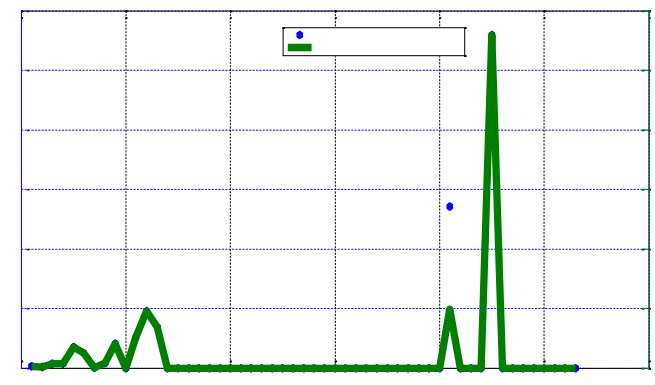

$$
\mathrm{Y} Z \rightarrow \mathrm{X}
$$

WHERE,

$X$-no. Of decision variables

Y- Generation by model -1

Z- Generation by model-2 
DOT(.) $\rightarrow$ Generation output for model-1

Green colour $\rightarrow$ Generation output for model-2.

\section{SUGGESTIONS AND FUTURE WORK:-}

\section{SUGGESTIONS:-}

$>$ Demand forecasting shall be made on the basis of updated information and the methods end-use and econometric analyses should be implemented.

$>$ Energy generation of the country shall need suitable composition so as to fix the energy balance by minimizing deforestation for energy consumption.

$>$ Incorporation of carbon trading in the decision of generation planning shall be better.

\section{FUTURE WORK:-}

$>$ The model can be modified by including transmission network system.

$>$ Evaluation of the economic and technical characteristics of the existing system of generating units.

$>$ The method of the combination of end-use and econometric analyses will be included in demand forecasting. This also adds more accuracy of the result to the model.

\section{CONCLUSIONS}

As we know that the current day situation is like demand is more than generation. The forecasted demand is increased because of urbanization, growth and transformation plan, economic and social developments.

The forecasted electrical energy demand done is increased averagely by $34.78 \%$ in scenario- 1 and by $31.98 \%$ in scenario- 2 yearly for the next 10 years. Comparing the forecasted demands by scenario- 1 and scenario- 2 with that of plan arecomparing the forecasted demands by scenario- 1 and scenario- 2 with that previous plan are averagely differed by $32 \%$ and $27 \%$ respectively yearly.

The investment and generation cost expended is an average of 0.0112 USD to produce a $\mathrm{kWh}$ electric energy in case of extra generation produced if carbon trade is included in generation expansion planning. It is very low compared to the cost of an average of 0.111 USD to produce a $\mathrm{kWh}$ electric energy by regular approach like model-2.

From the results of the models, the power generating plants having least unit cost $(\$ / M W h)$ is considered first for the decision of generation expansion. It has been shown that model-1 looks as if to be requiring higher cost. But, the investment unit cost for the extra generation in model-1 is highly reduced. And, model-1 needs lower levelized cost than in model-2.
To satisfy the electricity requirement, to maximize the benefits from the environmental preservation and to minimize the fuel to be imported for power plants, the right generation planning has been done by this thesis. Model-1 is the better model for optimal power generationexpansion planning
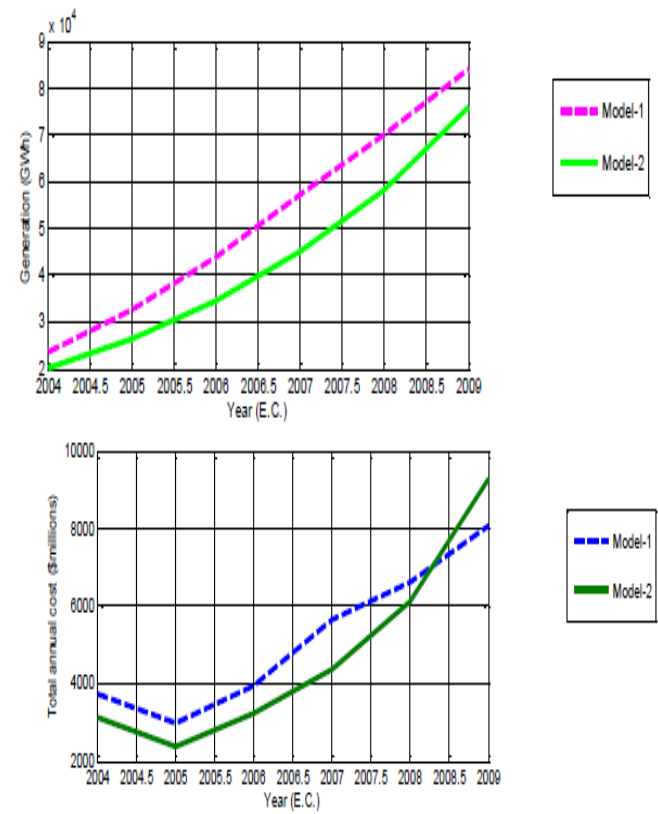

The above graphs shows The comparison of annual generation between model-1 and model-2; and The optimized total annual cost comparison between model-1 and model-2 in the period of time.

\section{REFERENCES}

[1] Angela S. Chuang, Felix Wu and PravinVaraiya: A GameTheoretic Model for Generation Expansion Planning: Problem Formulation and Numerical Comparisons, IEEE Transactions on Power Systems, Vol. 16, NO. 4, November 2001.

[2]Andras Ramos, Ignacio, J. PCrcz-Arriaga, and Juan Bogas: A Nonlinear Programming Approach to Optimal Static Generation Expansion Planning, IEEE Transactions on Power Systems, Vol. 4, No. 3, August 1989.

[3] E. Yu. Ivanova, N.I. Voropai, A. Orths, Z.A. Styczynski, Electric Power System Expansion Planning in Market Environment: Game Theoretical Approach, June 2002, http://www.pscc-

central.org/uploads/tx_ethpublications/s43p03.pdf

[4] Javier Contreras,FelixF.Wu: Coalition Formation In Transmission Expansion Planning.IEEE Transaction On Power System,Vol,14,No-3,August-1999

[5] Nikki Newham,University Of Canterbury,Power System Investment Planning Using Stochastic Dual Dynamic Programming ,April-2008.

[6] GirmawTeshager, Optimal Power generation Expansion for EEPCO-JULY-2011 